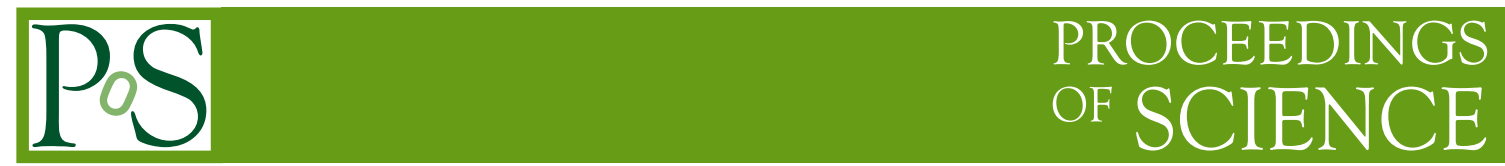

\title{
Schouten identities and the two-loop sunrise graph
}

\author{
Ettore Remiddi \\ DIFA, Università di Bologna and INFN, Sezione di Bologna, I-40126 Bologna, Italy \\ E-mail: Ettore.Remiddi@bo.infn.it
}

\section{Lorenzo Tancredi*}

Physik-Institut, Universität Zürich, Wintherturerstrasse 190, CH-8057 Zürich, Switzerland

E-mail: tancredi@physik.uzh.ch

\begin{abstract}
We introduce a new set of identities, dubbed Schouten identities, valid for Master Integrals in a fixed integer number of dimensions. We describe in detail how such identities can be used in order to simplify the differential equations for the four master integrals of the two-loop massive sunrise graph.
\end{abstract}

Loops and Legs in Quantum Field Theory

27 April 2014 - 02 May 2014

Weimar, Germany

\footnotetext{
${ }^{*}$ Speaker.
} 


\section{Introduction}

The Feynman integrals associated to the two-loop loop self-mass Feynman graph of Fig.(1), usually referred to as sunrise, have been widely studied in the literature within the framework of the integration by parts identities $[1,2]$, and it is by now well known that they can be expressed in terms of four Master Integrals (MIs), [3], which satisfy a system of four first-order coupled differential equations, [4] (equivalent to a single fourth-order differential equation for any of the Master Integrals).

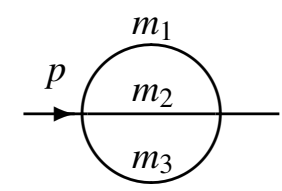

Figure 1: The two-loop sunrise.

In the Euclidean kinematics (so that $p^{2}$ is positive when spacelike) the MIs can be chosen as follows:

$$
\begin{aligned}
S\left(d ; p^{2}\right) & =\int \mathfrak{D}^{d} q_{1} \mathfrak{D}^{d} q_{2} \frac{1}{D_{1} D_{2} D_{3}}, \\
S_{1}\left(d ; p^{2}\right) & =-\frac{d}{d m_{1}^{2}} S\left(d ; p^{2}\right)=\int \mathfrak{D}^{d} q_{1} \mathfrak{D}^{d} q_{2} \frac{1}{D_{1}^{2} D_{2} D_{3}}, \\
S_{2}\left(d ; p^{2}\right) & =-\frac{d}{d m_{2}^{2}} S\left(d ; p^{2}\right)=\int \mathfrak{D}^{d} q_{1} \mathfrak{D}^{d} q_{2} \frac{1}{D_{1} D_{2}^{2} D_{3}}, \\
S_{3}\left(d ; p^{2}\right) & =-\frac{d}{d m_{3}^{2}} S\left(d ; p^{2}\right)=\int \mathfrak{D}^{d} q_{1} \mathfrak{D}^{d} q_{2} \frac{1}{D_{1} D_{2} D_{3}^{2}}
\end{aligned}
$$

The propagators are

$$
D_{1}=q_{1}^{2}+m_{1}^{2}, \quad D_{2}=q_{2}^{2}+m_{2}^{2}, \quad D_{3}=\left(p-q_{1}-q_{2}\right)^{2}+m_{3}^{2},
$$

and we define the loop integration measure as:

$$
\int \mathfrak{D}^{d} q=\frac{1}{C(d)} \int \frac{d^{d} q}{(2 \pi)^{d-2}}
$$

with

$$
C(d)=(4 \pi)^{(4-d) / 2} \Gamma\left(3-\frac{d}{2}\right) .
$$

With that definition the "double" tadpole reads

$$
T_{12}(d)=T\left(d ; m_{1}, m_{2}\right)=\int \mathfrak{D}^{d} q_{1} \mathfrak{D}^{d} q_{2} \frac{1}{D_{1} D_{2}}=\frac{\left(m_{1} m_{2}\right)^{d-2}}{(d-2)^{2}(d-4)^{2}},
$$

together with the similarly defined $T_{13}(d)=T\left(d ; m_{1}, m_{3}\right), T_{23}(d)=T\left(d ; m_{2}, m_{3}\right)$.

It has been shown [5], by using algebraic geometry arguments, that similarly to the equal mass case studied in detail in [6], in $d=2$ dimensions, the full scalar amplitude $S\left(d ; p^{2}\right)$ satisfies a 
second-order differential equation. The equation was then solved in [7] by suitably extending the method of [6]. Note that the analytic solution of the second-order differential equation is equivalent to the analytic knowledge of two (of the four) Master Integrals of the sunrise with different masses. Finally it has been recently shown that the finite piece of the scalar amplitude in $d=2$ can be expressed in terms of the newly introduced Elliptic polylogarithms [8, 9].

The problem of how to extend these results up to arbitrary orders in $(d-4)$, and in general, of how to systematically simplify the system of four coupled differential equations in order to obtain, in $d=2$, the second order equation derived in [5], remains. To this end in [10] we introduced a family of particular polynomials in the scalar products of the vectors occurring in the Feynman integrals, dubbed Schouten polynomials, which have the property of vanishing at some fixed integer value of the dimension $d$. By using those polynomials one can introduce an ad hoc set of amplitudes which vanish in a non trivial way at that value of $d$ (say at $d=N$ for definiteness). If those new amplitudes are expressed in terms of the "conventional" Master Integrals, their vanishing gives a set of relations between the latter, valid at $d=N$, which we call Schouten identities. One can then introduce a new set of Master Integrals including some of the independent amplitudes vanishing at $d=N$, write the system of differential equations satisfied by the new set of Master Integrals and expand them recursively in powers of $(d-N)$ around $d=N$. The newly system of equations takes then a simpler block structure around $d=N$.

The pattern is very general, and applies in principle to the integrals of any Feynman graph. Working out explicitly the case of the sunrise amplitudes at $d=2$ with different masses, one finds the existence of two independent Schouten identities, i.e. of two independent relations between the usual Master Integrals. This indicates that two of the four MIs are not independent in $d=2$ and therefore two out of the four differential equations can be decoupled in this limit. The other two equations remain coupled and, at order zero in $d=2$, give rise to the second-order differental equation found in [5]. At first-order in $(d-2)$ we find in particular two relatively simple equations for the first terms of the expansion of the two new MIs, in which the zeroth-orders of the two "conventional" MIs appear as non homogeneous known terms. This pattern can in principle be iterated up to any order in $(d-2)$. One can finally move from $d \approx 2$ to the physically more interesting $d \approx 4$ case by means of the Tarasov-Lee shifting relations [11, 12]; it is found that for obtaining the zeroth-order term in $(d-4)$ of all the four MIs (of the old or of the new set) at $d \approx 4$ one needs, besides the zeroth-order term in $(d-2)$ of the two "old" MIs at $d \approx 2$, also the first term in $(d-2)$ of the new MIs [10].

\section{Schouten identities for Master Integrals}

As it is well known, in any integer number of dimensions $d=N$ one cannot have more than $N$ linearly independent vectors. This piece of information can be used to derive new identities among MIs. Let us consider 2 linearly independent vectors $a_{\mu}, b_{\mu}$ in $d=2$ dimensions. Consider now the quantity

$$
\varepsilon(a, b)=\varepsilon_{\mu v} a^{\mu} b^{v}
$$

where $\varepsilon_{\mu v}$ is the Levi-Civita tensor with two indices, with $\varepsilon_{11}=\varepsilon_{22}=0$ and $\varepsilon_{12}=-\varepsilon_{21}=1$. By 
squaring Eq.(2.1) we get at once

$$
\varepsilon^{2}(a, b)=a^{2} b^{2}-(a \cdot b)^{2}
$$

So far all quantities are defined strictly in $d=2$ dimensions. As it is obvious by its very definition, if the dimension $d$ takes any non-vanishing integer value smaller than 2, the r.h.s of equation (2.1) vanishes, and so does the r.h.s of (2.2). We proceed then defining the Schouten polynomial $P_{2}(d ; a, b)$ as

$$
P_{2}(d ; a, b)=a^{2} b^{2}-(a \cdot b)^{2},
$$

where now all quantities are assumed to be defined in $d$-continuous dimensions. By its very definition the Schouten polynomial $P_{2}$ vanishes for $d<2$ :

$$
P_{2}(1 ; a, b)=0 .
$$

Following the very same procedure, given any triplet of vectors $a^{\mu}, b^{\mu}, c^{\mu}$ defined in $d=3$ dimensions, we consider the quantity

$$
\varepsilon(a, b, c)=\varepsilon_{\mu v \rho} a^{\mu} b^{v} c^{\rho}, \quad \text { with } \quad \varepsilon_{123}=1,
$$

and evaluate its square as

$$
\varepsilon(a, b, c)^{2}=a^{2} b^{2} c^{2}-a^{2}(b \cdot c)^{2}-b^{2}(a \cdot c)^{2}-c^{2}(a \cdot b)^{2}+2(a \cdot b)(b \cdot c)(a \cdot c),
$$

where, again, all quantities are to be thought for the moment strictly in $d=3$ dimensions. We define then the Schouten polynomial $P_{3}(d ; a, b, c)$ as:

$$
P_{3}(d ; a, b, c)=a^{2} b^{2} c^{2}-a^{2}(b \cdot c)^{2}-b^{2}(a \cdot c)^{2}-c^{2}(a \cdot b)^{2}+2(a \cdot b)(b \cdot c)(a \cdot c),
$$

where now the three vectors $a^{\mu}, b^{\mu}, c^{\mu}$ are to be interpreted as $d$-dimensional vectors. By construction, $P_{3}(d ; a, b, c)$ vanishes at $d=1$ and $d=2$ dimensions

$$
P_{3}(1 ; a, b, c)=P_{3}(2 ; a, b, c)=0 .
$$

Needless to say, the procedure can be easily iterated in any integer number of dimensions $N$, provided that one has $N$ independent vectors to start with. We note that the Schouten polynomial generated by a given set of vectors is nothing by their Gram determinant.

Obviously in physical applications we are interested mainly in the $d \rightarrow 4$ limit. In this sense one would naively expect that the only relevant Schouten polynomials are those which vanish in $d=1,2,3,4$ dimensions, and so built up starting from 5 different vectors. Nevertheless, by means of the Tarasov-Lee dimensional shifts $[11,12]$, one can reach $d=4$ from any different, even value of $d$. In this sense the $d=1$ Schouten polynomials (2.3), easily established for any Feynman amplitude in which at least 2 vectors occur, are of no practical use. The next simplest example are the Schouten polynomials in $d=2$ (2.6), which can in turn be built up for any Feynman amplitude with at least 3 independent vectors. 


\section{The Schouten identities for the two-loop massive sunrise graph}

Let us consider now the two-loop massive sunrise graph introduced above. The latter depends on three momenta $p, q_{1}, q_{2}$, we can therefore introduce the Schouten amplitudes defined, for arbitrary $d$, as

$$
Z\left(d ; n_{1}, n_{2}, n_{3}, p^{2}\right)=\int \mathfrak{D}^{d} q_{1} \mathfrak{D}^{d} q_{2} \frac{P_{3}\left(d ; p, q_{1}, q_{2}\right)}{D_{1}^{n_{1}} D_{2}^{n_{2}} D_{3}^{n_{3}}},
$$

where the $n_{i}$ are positive integer numbers. The convergence of the integrals, for a given value of $d$, depends of course on the powers $n_{i}$, as the Schouten polynomial in the numerator contributes always with four powers of the loop momenta $q_{1}$ and $q_{2}$. We are interested here in the $d=2$ case. If the Schouten amplitude is convergent at $d=2$, due to Eq.(2.7), it is also vanishing at $d=2$, i.e. $Z\left(2 ; n_{1}, n_{2}, n_{3}, p^{2}\right)=0$. Note that in the massive case all the integrals we are considering are i.r. finite, therefore the divergences can only be of u.v. nature. A generalization to the case where i.r. are present is currently under study.

On the other hand, the Schouten amplitudes Eq.(3.1) can be reduced in terms of the "conventional" MIs given in Eq.s(1.1), and using $Z\left(2 ; n_{1}, n_{2}, n_{3}, p^{2}\right)=0$ one can study their limiting values as $d \rightarrow 2$. One finds in particular that the Schouten amplitudes which provide non-trivial relations among the conventional MIs as $d \rightarrow 2$ are those with the minimal values of the $n_{i}$ needed to ensure the convergence. One finds for example:

$$
\begin{aligned}
Z_{2}\left(d ; p^{2}\right) & =Z\left(d ; 2,1,2, p^{2}\right) \\
& =\frac{(d-1)}{12}\left[-(d-2) p^{2}+(d-3)\left(m_{1}^{2}-2 m_{2}^{2}+m_{3}^{2}\right)\right] S\left(d ; p^{2}\right) \\
& +\frac{(d-1)}{12}\left(p^{2}+m_{1}^{2}-3 m_{2}^{2}+3 m_{3}^{2}\right) m_{1}^{2} S_{1}\left(d, p^{2}\right) \\
& -\frac{(d-1)}{6}\left(p^{2}+m_{2}^{2}\right) m_{2}^{2} S_{2}\left(d ; p^{2}\right) \\
& +\frac{(d-1)}{12}\left(p^{2}+3 m_{1}^{2}-3 m_{2}^{2}+m_{3}^{2}\right) m_{3}^{2} S_{3}\left(d ; p^{2}\right) \\
& +\frac{(d-1)(d-2)}{24}\left[T\left(d ; m_{1}, m_{2}\right)-2 T\left(d ; m_{1}, m_{3}\right)+T\left(d ; m_{2}, m_{3}\right)\right],
\end{aligned}
$$

and similarly for the other two permutations $Z_{1}\left(d ; p^{2}\right)=Z(d ; 1,2,2)$ and $Z_{3}\left(d ; p^{2}\right)=Z\left(d ; 2,2,1, p^{2}\right)$. Increasing the powers of the denominators one finds instead:

$$
\begin{aligned}
Z\left(d ; 2,2,2, p^{2}\right) & =-\frac{(d-1)(d-2)}{4} \\
& \times\left[(d-3) S\left(d ; p^{2}\right)+m_{1}^{2} S_{1}\left(d ; p^{2}\right)+m_{2}^{2} S_{2}\left(d ; p^{2}\right)+m_{3}^{2} S_{3}\left(d ; p^{2}\right)\right],
\end{aligned}
$$

which obviously does not give any information as $d \rightarrow 2$.

On the other hand, studying this limit for one of the $Z_{i}$, say the $Z_{2}\left(d ; p^{2}\right)$ defined above, one 
finds:

$$
\begin{aligned}
Z_{2}\left(2 ; p^{2}\right) & =-\frac{1}{12}\left(m_{1}^{2}-2 m_{2}^{2}+m_{3}^{2}\right) S\left(2 ; p^{2}\right) \\
& +\frac{1}{12}\left(p^{2}+m_{1}^{2}-3 m_{2}^{2}+3 m_{3}^{2}\right) m_{1}^{2} S_{1}\left(2, p^{2}\right) \\
& -\frac{1}{6}\left(p^{2}+m_{2}^{2}\right) m_{2}^{2} S_{2}\left(2 ; p^{2}\right) \\
& +\frac{1}{12}\left(p^{2}+3 m_{1}^{2}-3 m_{2}^{2}+m_{3}^{2}\right) m_{3}^{2} S_{3}\left(2 ; p^{2}\right) \\
& +\frac{1}{96} \ln \frac{m_{2}^{2}}{m_{1} m_{3}} \\
& =0
\end{aligned}
$$

and similarly for $Z_{1}\left(d ; p^{2}\right)$ and $Z_{3}\left(d ; p^{2}\right)$, which can be found in [10] and we don't write here for the sake of brevity. Summing the relations for the three $Z_{i}\left(d ; p^{2}\right)$ one finds

$$
Z_{1}\left(d ; p^{2}\right)+Z_{2}\left(d ; p^{2}\right)+Z_{3}\left(d ; p^{2}\right)=-\frac{(d-1)(d-2)}{4} p^{2} S\left(d ; p^{2}\right),
$$

which proves that in the limit $d \rightarrow 2$ only two of the $Z_{i}$ are linearly independent. This suggests to take as new MIs two of the conventional MIs plus two independent Schouten amplitudes. A possible choice is

$$
S\left(d ; p^{2}\right), \quad S_{1}\left(d ; p^{2}\right), \quad Z_{2}\left(d ; p^{2}\right), \quad Z_{3}\left(d ; p^{2}\right) .
$$

\section{The differential equations in the new basis}

The next step consists in deriving a new set of differential equations for the basis defined in Eq. (3.6). The explicit expressions are cumbersome and we refer to [10] for details, while we are interested here only in the general structure of these equations. They can be written as

$$
\begin{aligned}
\frac{d}{d p^{2}} S\left(d ; p^{2}\right) & =C_{00}\left(d ; p^{2}\right) S\left(d ; p^{2}\right)+C_{01}\left(d ; p^{2}\right) S_{1}\left(d ; p^{2}\right) \\
& +C_{02}\left(d ; p^{2}\right) Z_{2}\left(d ; p^{2}\right)+C_{03}\left(d ; p^{2}\right) Z_{3}\left(d ; p^{2}\right) \\
& +D_{01}\left(d ; p^{2}\right) T_{12}(d)+D_{02}\left(d ; p^{2}\right) T_{13}(d) \\
& +D_{03}\left(d ; p^{2}\right) T_{23}(d), \\
\frac{d}{d p^{2}} S_{1}\left(d ; p^{2}\right) & =C_{10}\left(d ; p^{2}\right) S\left(d ; p^{2}\right)+C_{11}\left(d ; p^{2}\right) S_{1}\left(d ; p^{2}\right) \\
& +C_{12}\left(d ; p^{2}\right) Z_{2}\left(d ; p^{2}\right)+C_{13}\left(d ; p^{2}\right) Z_{3}\left(d ; p^{2}\right) \\
& +D_{11}\left(d ; p^{2}\right) T_{12}(d)+D_{12}\left(d ; p^{2}\right) T_{13}(d) \\
& +D_{13}\left(d ; p^{2}\right) T_{23}(d),
\end{aligned}
$$




$$
\begin{aligned}
\frac{d}{d p^{2}} Z_{2}\left(d ; p^{2}\right)=(d-2) & {\left[C_{20}\left(d ; p^{2}\right) S\left(d ; p^{2}\right)+C_{21}\left(d ; p^{2}\right) S_{1}\left(d ; p^{2}\right)\right.} \\
& +C_{22}\left(d ; p^{2}\right) Z_{2}\left(d ; p^{2}\right)+C_{23}\left(d ; p^{2}\right) Z_{3}\left(d ; p^{2}\right) \\
& +D_{21}\left(d ; p^{2}\right) T_{12}(d)+D_{22}\left(d ; p^{2}\right) T_{13}(d) \\
& \left.+D_{23}\left(d ; p^{2}\right) T_{23}(d)\right], \\
\frac{d}{d p^{2}} Z_{3}\left(d ; p^{2}\right)=(d-2) & {\left[C_{30}\left(d ; p^{2}\right) S\left(d ; p^{2}\right)+C_{31}\left(d ; p^{2}\right) S_{1}\left(d ; p^{2}\right)\right.} \\
& +C_{32}\left(d ; p^{2}\right) Z_{2}\left(d ; p^{2}\right)+C_{33}\left(d ; p^{2}\right) Z_{3}\left(d ; p^{2}\right) \\
& +D_{31}\left(d ; p^{2}\right) T_{12}(d)+D_{32}\left(d ; p^{2}\right) T_{13}(d) \\
& \left.+D_{33}\left(d ; p^{2}\right) T_{23}(d)\right] .
\end{aligned}
$$

Recall that all coefficients $C_{i j}\left(d ; p^{2}\right)$ and $D_{i j}\left(d ; p^{2}\right)$ depend also on the three masses $m_{i}, i=$ $1,2,3$. Note moreover that they are all rational functions and do not develop any pole as $d \rightarrow 2$. Another crucial point is that Eq.s $(4.3,4.4)$ both have an explicit factor $(d-2)$ in the r.h.s, which implies that, order by order in $(d-2)$, these equations are decoupled from the corresponding order of Eq.s $(4.1,4.2)$ and can be therefore, at least in principle, solved by quadrature. Finally, since $Z_{2}\left(2 ; p^{2}\right)=Z_{3}\left(2 ; p^{2}\right)=0$, then at order zero in $(d-2)$ Eq.s $(4.1,4.2)$ form a system of two coupled differential equations, which can be rephrased as a second-order differential equation for $S\left(2 ; p^{2}\right)$ or, equivalently, for $S_{1}\left(2 ; p^{2}\right)$. Upon doing this one recovers for $S\left(2 ; p^{2}\right)$ precisely the differential equation obtained in [5]. The solution of this equation can be used to determine $S_{1}\left(2 ; p^{2}\right)$. These can then be plugged into Eq.s $(4.3,4.4)$ giving two decoupled first-order differential equations for the first orders in $(d-2)$ of the two new MIs, $Z_{2}^{(1)}\left(2 ; p^{2}\right), Z_{3}^{(1)}\left(2 ; p^{2}\right)$. This procedure can be iterated in principle up to any order [10].

\section{Conclusions and outlook}

We showed how the recently introduced Schouten identities can be applied to the case of the massive two-loop sunrise graph with different masses, finding that in $d=2$ dimensions only two of the four Master Integrals (MIs) are actually independent, so that the other two can be expressed as suitable linear combinations of the latter. In the general case of arbitrary dimension $d$ and different masses, the four MIs are known to fulfil a system of four first-order coupled differential equations in the external momentum transfer. The system can equivalently be re-phrased as a fourth-order differential equation for one of the MIs only.

Using these relations we introduced a new set of four independent MIs, valid for any number of dimensions $d$, whose property is that two of the newly defined integrals vanish identically in $d=2$. The new system of differential equations for this set of MIs takes then a simpler block form when expanded in $(d-2)$, showing that order by order in $(d-2)$ the scalar amplitude of the two-loop massive sunrise fulfils a second order differential equation. 
The applications of these identities to more complicated cases, also involving i.r. divergent amplitudes, is currently under study, and might constitute a promising tool for systematically decoupling systems of many coupled differential equations.

\section{References}

[1] K. Chetyrkin and F. Tkachov, Integration by Parts: The Algorithm to Calculate beta Functions in 4 Loops, Nucl.Phys. B192 (1981) 159-204.

[2] F. Tkachov, A Theorem on Analytical Calculability of Four Loop Renormalization Group Functions, Phys.Lett. B100 (1981) 65-68.

[3] O. Tarasov, Generalized recurrence relations for two loop propagator integrals with arbitrary masses, Nucl.Phys. B502 (1997) 455-482, [hep-ph / 9703319$].$

[4] M. Caffo, H. Czyz, S. Laporta, and E. Remiddi, The Master differential equations for the two loop sunrise selfmass amplitudes, Nuovo Cim. A111 (1998) 365-389, [hep-th/9805118].

[5] S. Mueller-Stach, S. Weinzierl, and R. Zayadeh, A Second-Order Differential Equation for the Two-Loop Sunrise Graph with Arbitrary Masses, Commun.Num.Theor.Phys. 6 (2012) 203-222, [arXiv:1112.4360].

[6] S. Laporta and E. Remiddi, Analytic treatment of the two loop equal mass sunrise graph, Nucl.Phys. B704 (2005) 349-386, [hep-ph / 0406160 ].

[7] L. Adams, C. Bogner, and S. Weinzierl, The two-loop sunrise graph with arbitrary masses, arXiv:1302.7004.

[8] S. Bloch and P. Vanhove, The elliptic dilogarithm for the sunset graph, arXiv:1309.5865.

[9] L. Adams, C. Bogner, and S. Weinzierl, The two-loop sunrise graph with arbitrary masses in terms of elliptic dilogarithms, arXiv:1405.5640.

[10] E. Remiddi and L. Tancredi, Schouten identities for Feynman graph amplitudes; the Master Integrals for the two-loop massive sunrise graph, arXiv:1311.3342.

[11] O. Tarasov, Connection between Feynman integrals having different values of the space-time dimension, Phys.Rev. D54 (1996) 6479-6490, [hep-th/ 9606018$].$

[12] R. Lee, Space-time dimensionality D as complex variable: Calculating loop integrals using dimensional recurrence relation and analytical properties with respect to D, Nucl.Phys. $\mathbf{B 8 3 0}$ (2010) 474-492, [arXiv:0911.0252]. 